\title{
Low-intensity physical activity is associated with reduced risk of incident type 2 diabetes in older adults: evidence from the English Longitudinal Study of Ageing
}

\author{
P. Demakakos $\cdot$ M. Hamer $\cdot$ E. Stamatakis $\cdot$ A. Steptoe
}

Received: 20 December 2009/Accepted: 13 April 2010/Published online: 22 May 2010

(C) Springer-Verlag 2010

\begin{abstract}
Aims/hypothesis We examined whether small amounts of low-intensity physical activity were associated with reduced risk of developing type 2 diabetes in a national sample of people aged 50 years and over.

Methods The sample comprised 7,466 individuals $(55.9 \%$ women) free from self-reported doctor-diagnosed diabetes and was prospectively followed for a mean of 45.3 months. Baseline self-reported physical activity was categorised as physical inactivity, low- and vigorous/moderate-intensity physical activity at least once a week. Cox proportional hazard regression was used to model the association between baseline physical activity and incident type 2 diabetes.

Results Vigorous/moderate-intensity physical activity at least once a week was associated with reduced risk of type 2 diabetes (HR 0.64, 95\% CI 0.43-0.95, $p=0.026$ ) but lowintensity physical activity at least once a week was not (HR $0.87,95 \%$ CI $0.58-1.30, p=0.497$ ) after adjustment for all covariates. However, age-stratified analysis showed that low-intensity physical activity at least once a week was associated with reduced risk of type 2 diabetes for those aged 70 years and over (HR 0.53, 95\% CI 0.28-1.02, $p=$ 0.059 ), but not for those aged 50 to 59 years (HR 1.09, $95 \%$ CI $0.52-2.29, p=0.828$ ) or those aged 60 to 69 years (HR 1.15, 95\% CI 0.55-2.41, $p=0.715$ ) after adjustment for all covariates.

Conclusions/interpretation Compared with physical inactivity, any type of physical activity was associated with
\end{abstract}

P. Demakakos $(\bowtie) \cdot$ M. Hamer $\cdot$ E. Stamatakis $\cdot$ A. Steptoe

Department of Epidemiology and Public Health,

University College London,

1-19 Torrington Place,

London WC1E 6BT, UK

e-mail: p.demakakos@ucl.ac.uk reduced risk of type 2 diabetes in adults aged 70 years and over, while in adults aged 50 to 69 years, physical activity needed to be vigorous/moderate in intensity to be associated with reduced risk of type 2 diabetes.

Keywords Elderly · Epidemiology · Longitudinal study Physical activity Type 2 diabetes
Abbreviations
CES-D Center for Epidemiological Studies-Depression
ELSA English Longitudinal Study of Ageing
HSE Health Survey for England
SES Socioeconomic status

\section{Introduction}

The prevalence of diabetes in England almost doubled between 1994 and 2006 in men and more than doubled in women; approximately $10 \%$ of adults in England aged 75 years and over have been diagnosed with the condition [1]. The current epidemic is largely related to lifestyle factors that are related to obesity and insulin resistance, such as diminished physical activity and increased energy and fat intake. Prospective cohort studies have consistently demonstrated inverse associations between physical activity and risk of type 2 diabetes $[2,3]$. The protective effect of physical activity becomes apparent even with moderateintensity exercise such as walking [3], and most studies report dose-response associations between physical activity and diabetes risk. The exact shape of this dose-response association remains uncertain though [4], and the difference in the reduction in risk between the least active and the next least active groups is not always significant [2]. There is a 
need to study this association further in diverse populations so as to improve the effectiveness of physical activity interventions to reduce diabetes in younger as well as in older populations [5].

Despite the large increase in the prevalence of diabetes in older adults over time [1], few studies have examined the association between physical activity and risk of diabetes in elderly cohorts. In the Diabetes Prevention Program, structured advice on activity and diet was more effective for reducing the risk of developing type 2 diabetes in the older participants (60-85 years) than in the younger participants (25-44 and 45-59 years) [6]. Similarly, in the Finnish Diabetes Prevention Study, it was in the oldest age group ( $>61$ years) that nutritional counselling and promotion of physical activity were proven most successful for preventing diabetes, while in the youngest age group ( $<51$ years) the difference in the risk of diabetes between the intervention and control groups was not significant [7]. Non-intervention data also show that physical activity is independently associated with a $26 \%$ reduction in the risk of incident diabetes over 10 years follow-up among adults aged 65 years and over [8]. Thus, physical activity may be particularly important for preventing diabetes in later life, although there is presently insufficient evidence to substantiate this.

We examined the association between physical activity and incident diabetes in a national sample of middle-aged and older people from the English Longitudinal Study of Ageing (ELSA). Because the minimum amount of physical activity needed to achieve a decrease in the risk of developing type 2 diabetes is not well established [2, 4, 5], we tested whether even small amounts of weekly low-intensity physical activity reduce the risk of developing type 2 diabetes in comparison with physical inactivity. Moreover, we explored whether age modifies the association between categories of physical activity and incident type 2 diabetes because there is evidence that this association is stronger in older rather than in younger populations [6].

\section{Methods}

Participants ELSA is a prospective cohort study of community-dwelling men and women aged 50 years and over. The baseline ELSA interview (wave 1) in 2002-2003 included 11,523 individuals aged 50 years and over who were recruited from households that had participated in the Health Survey for England (HSE) in 1998, 1999 and 2001. HSE is a health interview and examination survey that, each year, takes different nationally representative samples of households in England. Participants are contacted once every 2 years for the purposes of the ELSA interview and once every 4 years for the ELSA health examination. The first follow-up ELSA interview and the first ELSA health examination (wave 2) were in 2004-2005 and the second follow-up ELSA interview (wave 3) was in 2006-2007. Data from the HSE in 1998, 1999 and 2001, which predated the ELSA baseline and were available for the ELSA participants, are known as ELSA wave 0.

The analytical sample included 7,466 individuals $(55.9 \%$ women) who were aged 50 years and over and were free from self-reported doctor-diagnosed diabetes at baseline. Individuals who had been diagnosed with diabetes before the baseline $(n=877)$ along with individuals without longitudinal data $(n=2,078)$ or with missing data in any of the variables used in the analyses $(n=1,102)$ were not included in the analytical sample. Attrition after the baseline interview in our study was related to older age, male sex, lower education, elevated depressive symptoms and increased cardiovascular and noncardiovascular morbidity.

ELSA has been approved by various ethics committees, including the London Multicentre Research Ethics Committee, and informed consent has been obtained from all participants.

Assessment of diabetes The outcome measure was incident self-reported doctor-diagnosed diabetes (i.e. new cases of diabetes that were diagnosed after the baseline interview). All new cases of diabetes were treated as new cases of type 2 rather than type 1 diabetes because of the age of the participants (50 years or older). Because medical records were not available to confirm the diagnosis of diabetes and blood samples and data on use of diabetes medication were not collected at baseline, we used data on use of diabetes medication from ELSA wave 0 that were collected before the ELSA baseline (in HSE 1998, 1999 and 2001) to confirm diagnosis of diabetes in our participants. These data were available only for those of our participants who had had a nurse visit in ELSA wave $0 \quad(n=6,011$ out of 7,469 participants initially selected for inclusion in our study). Among the 6,011 participants with diabetes medication data from wave 0 , we identified only three cases that had been misclassified in our study as free from diabetes at baseline in 2002-2003, as they had been taking diabetes medication before 2002. We excluded these individuals from further analyses and assigned them to the group of prevalent cases of diabetes. This finding indicated that the impact of under-reporting of doctor-diagnosed diabetes on our analysis was minimal.

Measurement of physical activity Participants were asked how often they took part in three different types of physical activity: vigorous, moderate and low intensity. The response options were: more than once a week, once a week, one to three times a month and hardly ever/never. For the purposes of the analysis we derived a summary index of physical activity by summing responses to the three 
physical activity questions after they had been dichotomised around the frequency cut-point of once a week or more often. The derived summary index categorised physical activity as follows: (1) physical inactivity; (2) low-intensity but not vigorous/moderate-intensity physical activity at least once a week; and (3) vigorous/moderate-intensity physical activity at least once a week. The reliability and validity of our measure have not been assessed but a similar categorisation of physical activity has previously been shown to demonstrate excellent convergent validity in grading a plethora of biochemical and other risk factors, including BMI, triacylglycerols, fibrinogen and C-reactive protein in the same cohort [9].

Measurement of covariates Baseline sociodemographic, lifestyle and clinical characteristics of the sample were used as covariates. Age was measured in years (with all ages over 90 years being coded as 91). Sex, marital status (married/non-married), existence of long-standing illness or disability (yes/no), smoking status (never a smoker, exsmoker and current smoker) and frequency of alcohol consumption (daily or almost daily, once or twice a week, once or twice a month and almost never or never) were also assessed. Socioeconomic status (SES) was measured by quintiles of total non-pension household wealth (with household defined as a couple with any dependent child they may have) less debts owed by the household and by educational attainment (university degree or equivalent, some educational qualifications and no educational qualifications). Data on self-reported doctor-diagnosed cardiovascular morbidity (hypertension, angina, heart attack, congestive heart failure, heart murmur, abnormal heart rhythm and stroke) and self-reported doctor-diagnosed noncardiovascular morbidity (chronic lung disease, asthma, arthritis, osteoporosis, cancer/malignant tumour excluding minor skin cancers, Parkinson's disease, emotional/nervous/ psychiatric problems, Alzheimer's disease and dementia or other serious memory impairment) were also used. Depressive symptoms were assessed as the summary score of the eight-item Center for Epidemiological Studies-Depression (CES-D) scale dichotomised around the cut-point of four or more depressive symptoms [10]. Because anthropometric data were not collected at baseline (wave 1), we calculated BMI by using pre-ELSA data on height and weight that were collected by nurses during a clinical examination in ELSA wave 0.

Statistical analysis Baseline characteristics were analysed by physical activity categories and tests for trend were used. Follow-up time (in months) was calculated as the time elapsed between the date of baseline interview and the first of either the self-reported date of diagnosis of diabetes or the date of the last ELSA interview for the individual.
The prospective association between categories of baseline physical activity and incidence of type 2 diabetes was assessed using Cox proportional hazard regression. First, we estimated multivariable Cox proportional hazard regression models for the entire sample. Then, because evidence suggests that age may modify the effect of lifestyle interventions on preventing type 2 diabetes [6] and retirement may be a determinant of health [11], we estimated multivariable Cox proportional hazard models for three different age categories (50-59, 60-69 and $\geq 70$ years). The decision to perform age-stratified analysis, even though the interaction terms between physical activity and age categories were not statistically significant, was also made on the basis that the lack of statistically significant interaction does not imply that the association takes necessarily the same form in all age categories. The interaction terms between physical activity and sex and BMI were also not significant. In both analyses, the ageadjusted model was progressively adjusted for sex, marital status, SES, BMI, cardiovascular and non-cardiovascular comorbidities, health behaviours and depressive symptomatology. The estimates for the risk of developing type 2 diabetes in the Cox regression models are presented in the form of hazard ratios and $95 \% \mathrm{CI}$.

The proportional hazards assumption was confirmed by the use of graphical plots (log-negative log survival plots) and statistical tests (Schoenfeld residuals tests). We repeated our multivariable analysis after having removed all incident cases of diabetes that were diagnosed within the first 12 months after the baseline $(n=7,434)$ in order to test whether reverse causality might explain our results (baseline undiagnosed diabetes causing physical inactivity). Similarly, we repeated our multivariable analysis for only those participants without any limiting long-standing illness or disability $(n=5,186)$, because physical limitations and serious somatic health problems may influence the association between physical activity and type 2 diabetes. Further, we assessed the potential effect of missing data on our results by estimating an age-adjusted Cox regression model for a larger sample that included cases with missing data in any of the covariates ( $n=$ 8,470 ). Finally, we repeated our analysis using the average of BMI from wave 0 and wave 2 (as an estimate of BMI at wave 1) instead of BMI at wave $0(n=6,109)$, because wave 0 BMI may underestimate BMI at wave 1 and thus affect our results. The analysis was performed using STATA 9.2 and the level of statistical significance was $p \leq 0.05$.

\section{Results}

Physical inactivity was related to older age, not being married, lower SES, higher prevalence of cardiovascular 
and non-cardiovascular morbidity and long-standing illness/disability, elevated depressive symptoms, current smoking, non-use of alcohol and higher BMI (Table 1). Over almost 4 years of follow-up (45.3 months) there were 258 incident cases of doctor-diagnosed diabetes (Table 2). The Cox regression models that were estimated for the entire sample (Table 3) showed that the risk of developing type 2 diabetes was lower for participants who performed any type of physical activity at least once a week compared with those who were physically inactive. Vigorous/moderateintensity physical activity performed at least once a week was associated with reduced risk for diabetes after adjustment for

Table 1 Baseline characteristics of 7,466 men and women without prevalent type 2 diabetes by physical activity at baseline, English Longitudinal Study of Ageing, 2002-2003

\begin{tabular}{|c|c|c|c|c|}
\hline \multirow[t]{2}{*}{ Variable } & \multicolumn{3}{|c|}{ Physical activity performed at least once a week } & \multirow{2}{*}{$\begin{array}{l}p \text { value for } \\
\text { trend }\end{array}$} \\
\hline & None $(n=550)$ & $\begin{array}{l}\text { Low intensity } \\
(n=1,966)\end{array}$ & $\begin{array}{l}\text { Vigorous to moderate } \\
\text { intensity }(n=4,950)\end{array}$ & \\
\hline Age, mean (SD), years & $68.3(11.0)$ & $65.4(10.2)$ & $62.9(9.1)$ & $<0.001$ \\
\hline Sex, $n(\%)$ & & & & $<0.001$ \\
\hline Men & $252(45.8)$ & $717(36.5)$ & $2,323(46.9)$ & \\
\hline Women & $298(54.2)$ & $1,249(63.5)$ & $2,627(53.1)$ & \\
\hline Marital status, $n(\%)$ & & & & $<0.001$ \\
\hline Non-married & $237(43.1)$ & $744(37.8)$ & $1,442(29.1)$ & \\
\hline Married & $313(56.9)$ & $1,222(62.2)$ & $3,508(70.9)$ & \\
\hline Education, $n(\%)$ & & & & $<0.001$ \\
\hline Degree or equivalent & $38(6.9)$ & $143(7.3)$ & $732(14.8)$ & \\
\hline Some qualifications & $193(35.1)$ & $884(45.0)$ & $2,583(52.2)$ & \\
\hline No qualification & $319(58.0)$ & $939(47.7)$ & $1,635(33.0)$ & \\
\hline $\begin{array}{l}\text { Total net non-pension household wealth, } \\
\text { median (interquartile range), } £\end{array}$ & $71,085(163,094)$ & $102,000(179,200)$ & $163,376(223,450)$ & $<0.001$ \\
\hline Any long-standing illness or disability, $n(\%)$ & & & & $<0.001$ \\
\hline No & $145(26.4)$ & 785 (39.9) & $2,656(53.7)$ & \\
\hline Yes & $405(73.6)$ & $1,181(60.1)$ & $2,294(46.3)$ & \\
\hline Cardiovascular comorbidity $^{\mathrm{a}}, n(\%)$ & & & & $<0.001$ \\
\hline No comorbid cardiovascular disease & $234(42.5)$ & $966(49.1)$ & $2,894(58.5)$ & \\
\hline At least one comorbid cardiovascular disease & $316(57.5)$ & $1,000(50.9)$ & $2,056(41.5)$ & \\
\hline Other non-cardiovascular comorbidity ${ }^{\mathrm{b}}, n(\%)$ & & & & $<0.001$ \\
\hline No comorbid non-cardiovascular disease & $201(36.6)$ & $855(43.5)$ & $2,713(54.8)$ & \\
\hline At least one comorbid non-cardiovascular disease & $349(63.4)$ & $1,111(56.5)$ & $2,237(45.2)$ & \\
\hline $\begin{array}{l}\text { Depressive symptoms (measured by the } 8 \text {-item } \\
\text { CES-D scale), } n(\%)\end{array}$ & & & & $<0.001$ \\
\hline No or non-elevated depressive symptoms $($ CES-D score $<4)$ & $378(68.7)$ & $1,588(80.8)$ & $4,418(89.3)$ & \\
\hline Elevated depressive symptoms (CES-D score $\geq 4$ ) & $172(31.3)$ & $378(19.2)$ & $532(10.7)$ & \\
\hline Smoking status, $n(\%)$ & & & & $<0.001$ \\
\hline Never a smoker & $189(34.4)$ & $693(35.2)$ & $1,873(37.8)$ & \\
\hline Ex-smoker & $250(45.4)$ & $853(43.4)$ & $2,278(46.0)$ & \\
\hline Current smoker & $111(20.2)$ & $420(21.4)$ & 799 (16.2) & \\
\hline Frequency of alcohol consumption, $n(\%)$ & & & & $<0.001$ \\
\hline Daily or almost daily & $129(23.4)$ & $455(23.1)$ & $1,601(32.4)$ & \\
\hline Once or twice a week & $121(22.0)$ & $615(31.3)$ & $1,654(33.4)$ & \\
\hline Once or twice a month & $52(9.5)$ & $237(12.1)$ & $510(10.3)$ & \\
\hline Never or almost never & $248(45.1)$ & $659(33.5)$ & $1,185(23.9)$ & \\
\hline BMI, mean (SD), $\mathrm{kg} / \mathrm{m}^{2}$ & $28.4(5.3)$ & $28.0(4.7)$ & $27.1(4.2)$ & $<0.001$ \\
\hline
\end{tabular}

${ }^{a}$ Cardiovascular comorbidities included: hypertension, angina, heart attack, congestive heart failure, heart murmur, abnormal heart rhythm and stroke

${ }^{\mathrm{b}}$ Non-cardiovascular comorbidities included: chronic lung disease, asthma, arthritis, osteoporosis, cancer/malignant tumour excluding minor skin cancers, Parkinson's disease, emotional/nervous/psychiatric problems, Alzheimer's disease and dementia or other serious memory impairment 
Table 2 Incidence of type 2 diabetes by physical activity at baseline in 7,466 men and women, English Longitudinal Study of Ageing, 2002-2007

Physical activity performed at least once a week

\begin{tabular}{lll}
\hline $\begin{array}{l}\text { None } \\
(n=550)\end{array}$ & $\begin{array}{ll}\text { Low } \\
\text { intensity } \\
(n=1,966)\end{array}$ & $\begin{array}{l}\text { Vigorous/ } \\
\text { moderate } \\
\text { intensity } \\
(n=4,950)\end{array}$ \\
\end{tabular}

\begin{tabular}{lrrr}
\hline Incident cases, $n$ & 35 & 88 & 135 \\
Person-years & 1,987 & 7,319 & 18,848 \\
$\begin{array}{l}\text { Incidence rate of type } \\
\text { 2 diabetes per 1,000 } \\
\text { person-years }\end{array}$ & 17.6 & 12.0 & 7.2 \\
\hline
\end{tabular}

covariates (HR $0.41,95 \%$ CI $0.28-0.59, p<0.001$ and HR $0.64,95 \%$ CI $0.43-0.95, p=0.026$ in the age-adjusted and fully adjusted models, respectively). Low-intensity physical activity performed at least once a week was also associated with reduced diabetes risk but its effect was less strong and the association was not statistically significant (HR 0.68, 95\% CI $0.46-1.01, p=0.058$ and HR 0.87 , 95\% CI $0.58-$ $1.30, p=0.497$ in the age-adjusted and fully adjusted models, respectively). Of all covariates used in these models, SES and BMI had the strongest effects on the association.

A sensitivity analysis that did not include cases of diabetes that were diagnosed within 12 months after the baseline $(n=7,434)$ produced results similar to those of the main analysis (HR $0.82,95 \%$ CI $0.54-1.25, p=0.359$ and HR 0.59 , 95\% CI $0.39-0.89, p=0.011$ for the low- and vigorous/moderate-intensity physical activity categories, respectively, in the fully adjusted model). The point estimates of the sensitivity analysis that was based on 5,186 individuals without self-reported limiting longstanding illness or disability were similar to those from the main analysis but were not statistically significant (HR $0.89,95 \%$ CI $0.44-1.80, p=0.751$ and $\mathrm{HR} 0.64,95 \% \mathrm{CI}$ $0.33-1.24, p=0.184$ for the low- and vigorous/moderateintensity physical activity categories, respectively, in the fully adjusted model). The age-adjusted model estimated for a larger sample that included cases with missing values $(n=8,470)$ produced results similar to those from the main analysis (HR 0.66, 95\% CI 0.46-0.94, $p=0.20$ and HR $0.39,95 \%$ CI $0.28-0.55, p<0.001$ for the low- and vigorous/moderate-intensity physical activity categories, respectively). Cox regression models that used the average of BMI from waves 0 and 2 instead of BMI at wave 0 ( $n=$ 6,109 ) produced almost identical results (HR 0.69, 95\% CI $0.45-1.07, p=0.101$ and HR $0.53,95 \%$ CI $0.35-0.81, p=$ 0.003 for the low- and vigorous/moderate-intensity physical activity categories, respectively, in the fully adjusted model) to those of models using BMI at wave 0 that were estimated for the same population $(n=6,109$; HR $0.69,95 \%$ CI $0.45-1.07, p=0.100$ and HR $0.52,95 \%$ CI $0.34-0.80$, $p=0.002$ for the low- and vigorous/moderate-intensity physical activity categories, respectively, in the fully adjusted model).

The age-stratified Cox regression models (Figs 1 and 2) showed that there were significant age differences in the association between physical activity and type 2 diabetes. Low-intensity physical activity performed at least once a

Table 3 HRs (95\% CI) of type 2 diabetes by physical activity at baseline in 7,466 men and women, English Longitudinal Study of Ageing, 2002-2007

Cox regression model

Physical activity performed at least once a week

\begin{tabular}{lllll}
\hline $\begin{array}{l}\text { None } \\
(n=550)\end{array}$ & Low intensity & $p$ & Vigorous/ & $p$ \\
& $(n=1,966)$ & value & $\begin{array}{l}\text { moderate } \\
\text { intensity } \\
(n=4,950)\end{array}$ & value \\
& & & & \\
\hline
\end{tabular}

Model 1: adjusted for age

Model 2: adjusted for age, age-squared, sex and marital status

Model 3: adjusted as for model 2 and for educational attainment and total household wealth

Model 4: adjusted as for model 3 and for BMI

Model 5: adjusted as for model 4 and for self-reported long-standing illness/disability and cardiovascular ${ }^{\mathrm{a}}$ and non-cardiovascular comorbidities $^{\mathrm{b}}$

Model 6: adjusted as for model 5 and for health behaviours

Model 7: adjusted as for model 6 and for elevated depressive symptoms

$\begin{array}{lllll}\text { Reference } & 0.68(0.46-1.01) & 0.058 & 0.41(0.28-0.59) & <0.001 \\ \text { Reference } & 0.69(0.47-1.03) & 0.066 & 0.40(0.27-0.58) & <0.001 \\ \text { Reference } & 0.76(0.51-1.13) & 0.170 & 0.49(0.33-0.71) & <0.001 \\ & & & & \\ \text { Reference } & 0.83(0.56-1.23) & 0.339 & 0.57(0.39-0.84) & 0.005 \\ \text { Reference } & 0.84(0.56-1.24) & 0.376 & 0.61(0.41-0.90) & 0.012 \\ & & & & \\ \text { Reference } & 0.84(0.57-1.25) & 0.391 & 0.62(0.42-0.91) & 0.015 \\ \text { Reference } & 0.87(0.58-1.30) & 0.497 & 0.64(0.43-0.95) & 0.026\end{array}$

${ }^{a}$ Cardiovascular comorbidities included: hypertension, angina, heart attack, congestive heart failure, heart murmur, abnormal heart rhythm and stroke

${ }^{\mathrm{b}}$ Non-cardiovascular comorbidities included: chronic lung disease, asthma, arthritis, osteoporosis, cancer/malignant tumour excluding minor skin cancers, Parkinson's disease, emotional/nervous/psychiatric problems, Alzheimer's disease and dementia or other serious memory impairment 


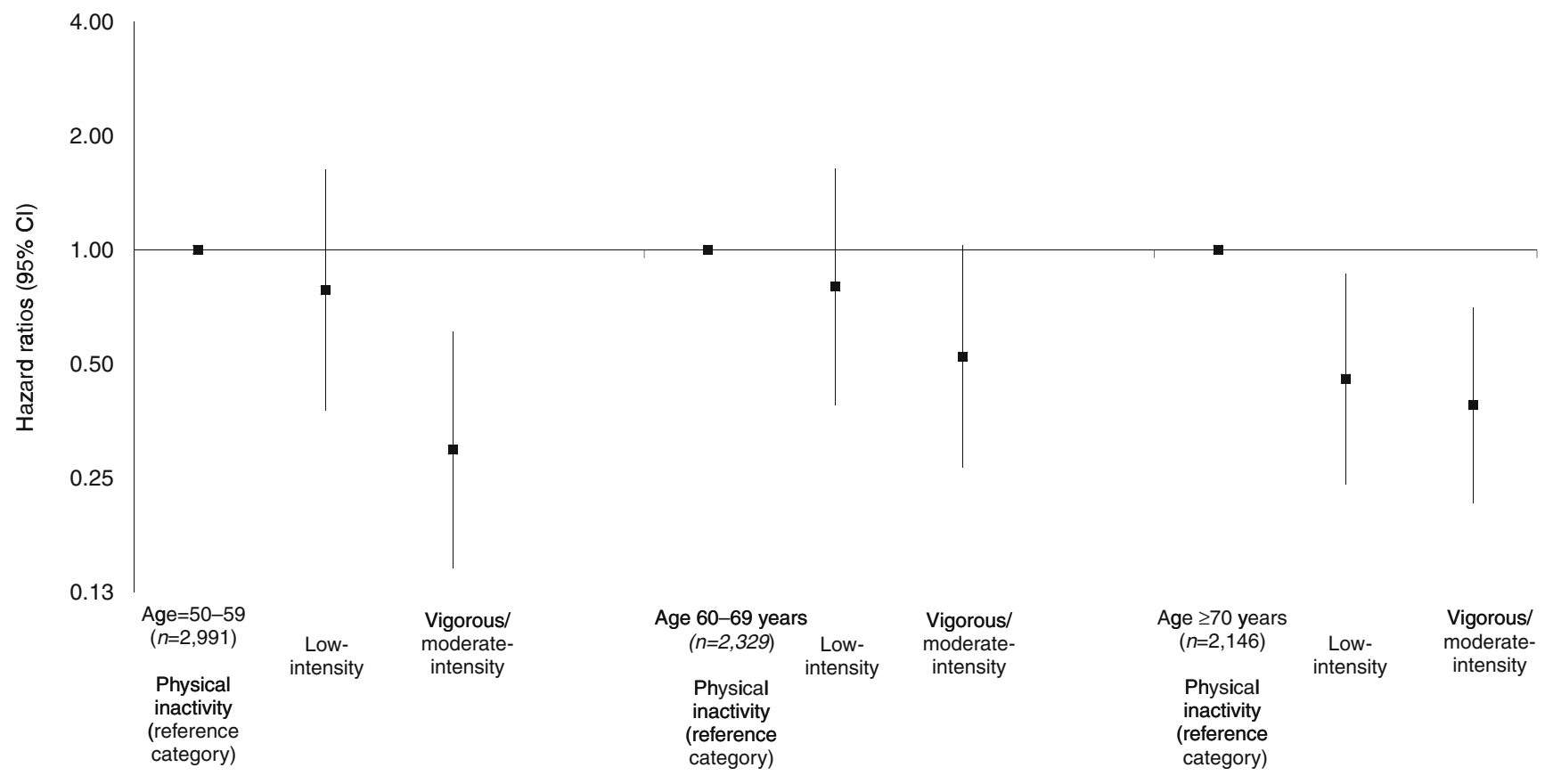

Fig. 1 Age-adjusted HRs (95\% CI) for type 2 diabetes by physical activity performed at least once a week at baseline and age category in 7,466 men and women, English Longitudinal Study of Ageing, 2002-2007

week was not related to diabetes risk in those aged 50 to 69 years, while it was clearly associated with reduced diabetes risk in those aged 70 years and over. In the youngest category (50 to 59 years), the age-adjusted HRs were $0.78(95 \% \mathrm{CI} 0.38-1.62, p=0.509)$ and $0.30(95 \% \mathrm{CI}$ $0.15-0.61, p=0.001)$ for the low- and vigorous/moderate- intensity physical activity categories, respectively. Additional adjustment for all other covariates except BMI reduced the strength of the association (HR 1.07, 95\% CI $0.51-2.26, p=0.856$ for the low-intensity category and HR $0.50,95 \%$ CI $0.24-1.06, p=0.072$ for the vigorous/ moderate-intensity), while further adjustment for BMI did

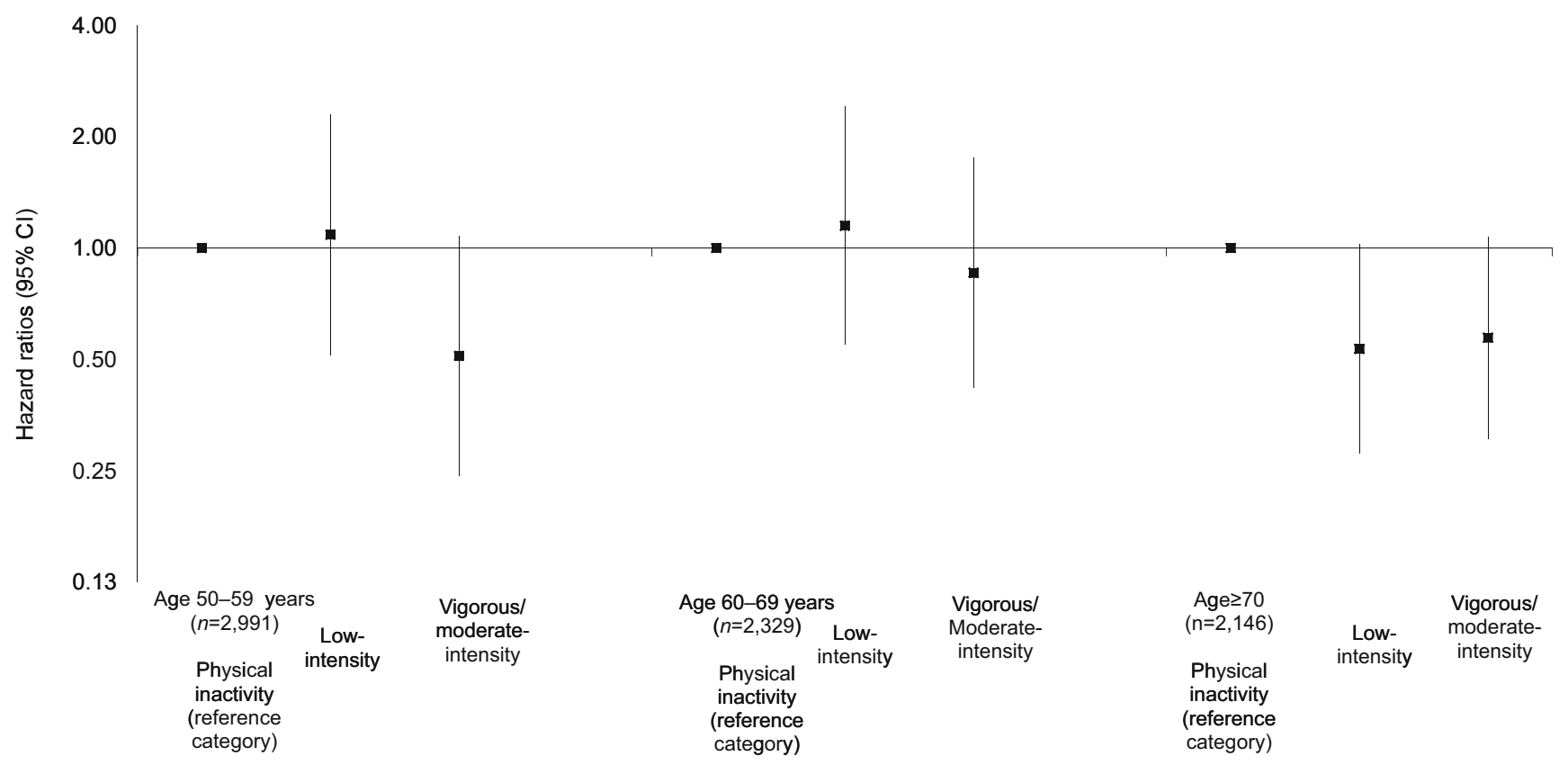

Fig. 2 Fully adjusted HRs $(95 \%$ CI) for type 2 diabetes by physical activity performed at least once a week at baseline and age category in 7,466 men and women, English Longitudinal Study of Ageing, 2002-2007 
not affect it (HR 1.09, 95\% CI $0.52-2.29, p=0.828$ for the low-intensity category and HR $0.51,95 \%$ CI $0.24-1.08, p=$ 0.078 for the vigorous/moderate-intensity). In the intermediate age category (60 to 69 years), the age-adjusted HR was $0.80(95 \%$ CI $0.39-1.64, p=0.541)$ for those performing low-intensity physical activity and $0.52(95 \%$ CI $0.27-1.03, p=0.060$ ) for those performing vigorous/ moderate-intensity physical activity. The respective estimates were: $1.06(95 \%$ CI $0.51-2.23, p=0.876)$ and 0.75 $(95 \%$ CI $0.37-1.54, p=0.437)$ in models adjusted for all covariates except BMI; and 1.15 (95\% CI 0.55-2.41, $p=$ $0.715)$ and $0.86(95 \%$ CI $0.42-1.76, p=0.673)$ in the fully adjusted models. In the oldest category ( $\geq 70$ years) the ageadjusted HRs were $0.46(95 \%$ CI $0.24-0.87, p=0.017)$ and 0.39 (95\% CI $0.22-0.70, p=0.002)$ for the low- and vigorous/moderate-intensity physical activity categories, respectively; additional adjustment for all other covariates except BMI explained some of this association (HR 0.53, 95\% CI $0.27-1.01, p=0.052$ and HR $0.54,95 \%$ CI 0.29 $1.01, p=0.053$ ). Further adjustment for BMI did not greatly affect the association (HR $0.53,95 \%$ CI $0.28-1.02, p=$ 0.059 and HR $0.57,95 \%$ CI $0.31-1.07, p=0.082$ for the low- and vigorous/moderate-intensity physical activity categories, respectively).

\section{Discussion}

In a national sample of men and women aged 50 years and over, physical activity was associated with a decreased risk of developing type 2 diabetes over approximately 4 years of follow-up, irrespective of adjustment for potential confounders such as obesity and SES. This finding is largely consistent with previous work $[2,3]$. The present study, however, adds to what is already known by showing that there are significant age differences in the association between physical activity and incident type 2 diabetes. In early old age (50 to 69 years) it was vigorous/moderate- but not low-intensity physical activity that conferred protection against diabetes, while at older ages ( $\geq 70$ years) any type of physical activity was associated with reduced diabetes risk compared with physical inactivity and no dose-response association was observed.

A number of strengths and weaknesses of our study need to be recognised. A major advantage of this study is the use of a large national sample of community-dwelling men and women. Another strength is the detailed assessment of baseline SES and cardiovascular and non-cardiovascular comorbidities which, together with the use of objectively measured BMI, allowed for an adequate adjustment in our models. The rigorous longitudinal design of ELSA allowed for a proper prospective exploration of the association between physical activity and diabetes. A further strength is the sensitivity analyses we performed, which showed that neither missing data nor the presence of limiting longstanding illness or disability could explain our findings (results in the latter analysis were not significant, most probably because of the lack of statistical power).

A major limitation is the use of a crude non-validated self-reported physical activity measure that did not allow for a proper examination of dose-response issues. For this reason and because self-reported physical activity in older adults has been shown to overestimate actual activity and underestimate the true effects of it on mortality in older adults [12], our study may have considerably underestimated the strength of associations between physical activity and diabetes. The use of self-reported diabetes data is another limitation of our work. Although we examined our self-reported diabetes data against information on past use of diabetes medication and performed a sensitivity analysis that excluded incident cases of diabetes that were diagnosed within the first 12 months after the baseline, we cannot completely rule out the possibility of reverse causality (undiagnosed diabetes causing physical inactivity at baseline). However, recent findings showing that there was overall good agreement (81.5\%) between self-reported doctor-diagnosed diabetes and measured fasting blood glucose in ELSA wave 2 [13] constitute further evidence that the effect of undiagnosed diabetes on our results, if any, is not expected to be great. Finally, the attrition that ELSA has suffered must also be considered. Our results may provide a conservative account of the association between physical activity and the risk of developing diabetes because it was mostly the less-educated participants who dropped out from ELSA. These individuals were probably less active (see Table 1) and therefore their risk for incident diabetes may have been higher.

There is, at present, a paucity of studies examining the association between physical activity and incidence of diabetes in adults aged 70 years and older. In the Cardiovascular Health Study of adults aged 65 years or older, physical activity was independently associated with a $26 \%$ reduction in diabetes risk over 10 years' follow-up [8]. A recent study of mortality in very old individuals found that smaller amounts of less intense physical activity than the recommended dose of $30 \mathrm{~min}$ of moderate-intensity physical activity per day for 5 days a week was associated with greater survival compared with physical inactivity [14]. Another recent study of mortality in adults 70 years old or older, which measured energy expenditure in the most objective way by using the doubly labelled water method, similarly suggested that any energy-expending activity may lower the risk of mortality among older adults [12]. Thus, our finding that relatively small amounts of low-intensity physical activity confer an advantage against developing type 2 diabetes among those aged 70 years and 
over is largely consistent with existing work, and indicates that any physical activity might provide health benefits in this age group. We also found that in this age group the association between physical activity and incident diabetes was not dose-dependent (this was also observed among those aged 65 to 69 years [data not shown]). To our knowledge no other study of older people has previously reported the lack of a dose-response association between physical activity and diabetes. Studies of mortality among those aged 70 and over have provided conflicting evidence on this issue as one study suggested that there was no clear dose-dependent effect of physical activity on the mortality risk and that the major difference in the risk was between sedentary and active older adults [14], while another study reported the existence of a clear dose-dependent association between physical activity and mortality [12]. The lack of a dose-response association is, of course, dependent on the cut-points we used to derive our physical activity measure, and therefore it should be considered in the light of a recent review on the dose-response relationship between physical activity and cardiovascular morbidity and mortality in older people [15]. This concluded that the nature of this relationship remains unclear because of the very broad range of dose-response schemes used in epidemiological surveys.

In the youngest age group (50 to 59 years) we found that vigorous/moderate-intensity physical activity was related to a $49 \%$ lower risk of developing type 2 diabetes after adjustment for all covariates. This finding accords with a meta-analytical review of ten cohort studies showing that moderate-intensity physical activity was associated with, on average, a $30 \%$ reduction in risk of diabetes [3]. The marginal lack of statistical significance in this association as well as in those involving participants aged 70 years and over probably reflects the lack of statistical power and thus is not per se greatly informative about the association examined. Our finding that low-intensity physical activity was not related to diabetes risk in this age group suggests that in adults aged 50 to 59 years, physical activity needs to be moderate or vigorous to confer an advantage against the risk of diabetes. It is also in agreement with a recent meta-analysis suggesting that it is physical activity above the level of least activity that appears to lower the risk of diabetes [2]. In the intermediate age group (60 to 69 years), the results were comparable with those of the youngest age group, suggesting that lowintensity physical activity was not related to a lower risk of developing diabetes compared with physical inactivity and underlying the need for vigorous physical activity to prevent diabetes in this age group.

Of all the covariates assessed in the analysis, only SES and obesity were found to affect the association, though they did not fully account for the results. Previous studies have shown that physical activity remained significantly related to incident diabetes after adjustment for a single measure of SES (typically education [16-18] but also occupational class [19]). Our study has accounted in a fuller way for SES by adjusting models for both education and total wealth, which is one of the best measures of SES for older people [20], and thus has more convincingly shown that the significant association between physical activity and incident diabetes is not an artefact of residual confounding because of inadequate adjustment for SES.

Obesity is a risk factor for diabetes that is known to interact with physical inactivity [21]. Our study, as previous studies [22, 23], found that obesity partly but not fully explained the association between physical activity and diabetes, and therefore provides support to suggestions that it is not only through weight regulation that physical activity reduces the risk for diabetes [4]. As weight loss is not the only mechanism through which physical activity is associated with type 2 diabetes, it is important to establish what other non-obesity-related biological mechanisms may play a role. Structural and biochemical changes in skeletal muscle and systemic changes such as improved hormonal homeostasis and improvements in dyslipidaemia and inflammation [4, 5, 24-26] may be such mechanisms. Other studies have also examined the association between acute exercise and rapid and pronounced induction of hepatic metabolic enzymes and regulators of metabolism or transcription, such as insulin receptor substrate in animals [27] and the role of proteins that regulate translocation of the glucose transporter isoform GLUT-4 such as Akt substrate [28].

In conclusion, our study shows that in early old age (50 to 69 years) it is vigorous/moderate-intensity physical activity, but not low-intensity physical activity, that is associated with reduced risk of type 2 diabetes, while at older ages ( $\geq 70$ years) any type of physical activity is associated with reduced risk of type 2 diabetes compared with physical inactivity. According to these results, physical activity recommendations for adults aged 70 years and over should encourage participation in any physical activity.

Acknowledgements The data were made available through the UK Data Archive. The English Longitudinal Study of Ageing was developed by a team of researchers based at University College London, the Institute of Fiscal Studies and the National Centre for Social Research. The funding is provided by the National Institute on Aging in the United States (grants 2RO1AG7644-01A1 and 2RO1AG017644) and a consortium of UK government departments coordinated by the Office for National Statistics. The developers and funders of ELSA and the Archive do not bear any responsibility for the analyses or interpretations presented here.

Duality of interest The authors declare that there is no duality of interest associated with this manuscript. 


\section{References}

1. Shelton N (2008) Diabetes. In: Craig R, Mindell J (eds) Health Survey for England 2006. Cardiovascular disease and risk factors in adults. The Information Centre, London, pp 63-84

2. Gill JM, Cooper AR (2008) Physical activity and prevention of type 2 diabetes mellitus. Sports Med 38:807-824

3. Jeon CY, Lokken RP, Hu FB, van Dam RM (2007) Physical activity of moderate intensity and risk of type 2 diabetes: a systematic review. Diabetes Care 30:744-752

4. Bassuk SS, Manson JE (2005) Epidemiological evidence for the role of physical activity in reducing risk of type 2 diabetes and cardiovascular disease. J Appl Physiol 99:1193-1204

5. LaMonte MJ, Blair SN, Church TS (2005) Physical activity and diabetes prevention. J Appl Physiol 99:1205-1213

6. Crandall J, Schade D, Ma Y et al (2006) The influence of age on the effects of lifestyle modification and metformin in prevention of diabetes. J Gerontol A Biol Sci Med Sci 61:1075-1081

7. Lindstrom J, Peltonen M, Eriksson JG et al (2008) Determinants for the effectiveness of lifestyle intervention in the Finnish Diabetes Prevention Study. Diabetes Care 31:857-862

8. Mozaffarian D, Kamineni A, Carnethon M, Djousse L, Mukamal KJ, Siscovick D (2009) Lifestyle risk factors and new-onset diabetes mellitus in older adults: the Cardiovascular Health Study. Arch Intern Med 169:798-807

9. Hamer M, Molloy GJ, de Oliveira C, Demakakos P (2009) Leisure time physical activity, risk of depressive symptoms, and inflammatory mediators: the English Longitudinal Study of Ageing. Psychoneuroendocrinology 34:1050-1055

10. Steffick DE, the HRS Health Working Group (2000) Documentation of affective functioning measures in the Health and Retirement Study. HRS/AHEAD Documentation Report DR005. Available from http://hrsonline.isr.umich.edu/sitedocs/userg/ dr-005.pdf, accessed 18 March 2010

11. Westerlund H, Kivimaki M, Singh-Manoux A et al (2009) Selfrated health before and after retirement in France (GAZEL): a cohort study. Lancet 374:1889-1896

12. Manini TM, Everhart JE, Patel KV et al (2006) Daily activity energy expenditure and mortality among older adults. JAMA 296:171-179

13. Pierce MB, Zaninotto P, Steel N, Mindell J (2009) Undiagnosed diabetes-data from the English longitudinal study of ageing. Diabet Med 26:679-685

14. Stessman J, Hammerman-Rozenberg R, Cohen A, Ein-Mor E, Jacobs JM (2009) Physical activity, function, and longevity among the very old. Arch Intern Med 169:1476-1483
15. Meyer K, Stolz C, Rott C, Laederach-Hofmann K (2009) Physical activity assessment and health outcomes in old age: how valid are dose-response relationships in epidemiologic studies? Eur Rev Aging Phys Activ 6:7-17

16. Hsia J, Wu L, Allen C et al (2005) Physical activity and diabetes risk in postmenopausal women. Am J Prev Med 28:19-25

17. Folsom AR, Kushi LH, Hong CP (2000) Physical activity and incident diabetes mellitus in postmenopausal women. Am J Public Health 90:134-138

18. Hu G, Qiao Q, Silventoinen K et al (2003) Occupational, commuting, and leisure-time physical activity in relation to risk for type 2 diabetes in middle-aged Finnish men and women. Diabetologia 46:322-329

19. Wannamethee SG, Shaper AG, Alberti KG (2000) Physical activity, metabolic factors, and the incidence of coronary heart disease and type 2 diabetes. Arch Intern Med 160:2108-2116

20. Demakakos P, Nazroo J, Breeze E, Marmot M (2008) Socioeconomic status and health: the role of subjective social status. Soc Sci Med 67:330-340

21. Qin L, Knol MJ, Corpeleijn E, Stolk RP (2010) Does physical activity modify the risk of obesity for type 2 diabetes: a review of epidemiological data. Eur J Epidemiol 25:5-12

22. Rana JS, Li TY, Manson JE, Hu FB (2007) Adiposity compared with physical inactivity and risk of type 2 diabetes in women. Diabetes Care 30:53-58

23. Weinstein AR, Sesso HD, Lee IM et al (2004) Relationship of physical activity vs body mass index with type 2 diabetes in women. JAMA 292:1188-1194

24. Ekelund U, Franks PW, Sharp S, Brage S, Wareham NJ (2007) Increase in physical activity energy expenditure is associated with reduced metabolic risk independent of change in fatness and fitness. Diabetes Care 30:2101-2106

25. Duncan GE, Perri MG, Theriaque DW, Hutson AD, Eckel RH, Stacpoole PW (2003) Exercise training, without weight loss, increases insulin sensitivity and postheparin plasma lipase activity in previously sedentary adults. Diabetes Care 26:557-562

26. Venables MC, Jeukendrup AE (2008) Endurance training and obesity: effect on substrate metabolism and insulin sensitivity. Med Sci Sports Exerc 40:495-502

27. Hoene M, Lehmann R, Hennige AM et al (2009) Acute regulation of metabolic genes and insulin receptor substrates in the liver of mice by one single bout of treadmill exercise. J Physiol 587:241252

28. Deshmukh AS, Hawley JA, Zierath JR (2008) Exercise-induced phospho-proteins in skeletal muscle. Int J Obes (Lond) 32(Suppl 4):S18-S23 